\title{
Residual currents generated from vacuum by an electric field pulse in 2+1 dimensional QED models
}

\author{
S.A. Smolyansky ${ }^{1}{ }^{\star}$, D.V. Churochkin ${ }^{1}$, V.V. Dmitriev ${ }^{1}$, A.D. Panferov ${ }^{1}$, and B. Kämpfer ${ }^{2}$ \\ ${ }^{1}$ Saratov State University, RU-410026, Saratov, Russia \\ ${ }^{2}$ Helmholtz-Zentrum Dresden-Rossendolf, D-01314, Dresden, Germany
}

\begin{abstract}
In the framework of strong field QED, the generation of a residual alternating polarization current is demonstrated, which remains after switching off an external field pulse. This effect is stipulated by inertial properties of the physical vacuum. In the standard vacuum $D=2+1 \mathrm{QED}$, this current is rapidly damped fast but can be available, apparently, for observation in the graphene, where the Fermi velocity $v_{F} \ll c$ plays an analogous role as the light velocity.
\end{abstract}

\section{Introduction}

We address the question whether there are residual electric currents which remain after the strong electromagnetic impulse passage. The total current is composed of the conductivity and polarization currents, and the residual conductivity current is constant. However, surprisingly, the residual polarization current turns out to be fast oscillating and damped. This is a peculiar inertial effect of the vacuum oscillations excited by an external field. Thus, according to this prediction, some alternate component will appear in the total residual current.

We will consider this effect in a rather general case for different variants of spinor QED models in the external spatially-homogeneous, time-dependent electric field where the spin effects can be separated from the basic effect of vacuum creation of the electron-positron plasma (EPP). We consider two such systems: $D=2+1$ massless QED and graphene. The last model will be considered rather in detail. In conclusion, we discuss a manifestation of this effect in some other systems.

The discussed effect has also another interesting feature in the case of models with a running coupling constant. It turns out that the polarization current is dominating in the area of small coupling constant. However, this situation turns into the opposite for a large coupling constant, as in some QCD models.

We are going to consider firstly a general kinetic theory which unifies the mentioned QED models (Sect. 2). Such an analysis establishes the necessary prerequisites for a discussion of the residual currents. After that, as a concrete example, the residual currents will be considered in graphene on the basic of a nonperturbative kinetic approach (Sect. 3). A discussion of the effect will be given in Sect. 4.

^e-mail: smol@sgu.ru 


\section{Kinetic theory}

The kinetic equation (KE) for the description of the EPP vacuum creation in a strong homogeneous time dependent electric field can be cast into the form of an integro-differential equation for several QED models at once [1]

$$
\dot{f}(t)=\frac{1}{2} \lambda(t) \int_{t_{0}}^{t} d t^{\prime} \lambda\left(t^{\prime}\right)\left[1-2 f\left(t^{\prime}\right)\right] \cos \left[2 \int_{t^{\prime}}^{t} d \tau \varepsilon(\vec{p}, \tau)\right] .
$$

The distribution function is defined in the momentum space, $f(t)=f(\vec{p}, t)$. Quasienergy $\varepsilon(\vec{p}, t)$ and the amplitude of the vacuum transition $\lambda(\vec{p}, t)$ have different form for different QED models.

Firstly, the KE (1) was obtained in $D=3+1$ QED for a linearly polarized electric field in the framework of the quasiparticle representation [2,3]. It is easy to show that the KE conserves its form in the two above-mentioned cases of $D=2+1$ massless QED with a general Hamiltonian. In the case of graphene, it corresponds to a low-energy model of excitations around the $\mathrm{K}$ point in the Brillouin zone for clean graphene (e.g., [4]), Thus,

$$
H(t)=V \int d^{2} x \Psi^{\dagger}(x) \hat{\vec{P}} \vec{\sigma} \Psi(x)
$$

where $\hat{P}_{k}=-i \hbar \nabla_{k}-(e / c) A_{k}(t), \quad k=1,2, A_{k}(t)=\left(0, A^{1}(t), A^{2}(t), 0\right)$ in the Hamilton gauge; $\sigma_{k}$ is the Pauli matrix; $V=c$ in the vacuum QED and $V=v_{F} \simeq 10^{8} \mathrm{~cm} / \mathrm{s}$ is the Fermi velocity in graphene. The $\mathrm{KE}$ (1) conserves its form also in the tight-binding model of nearest neighbor interactions [5]. Here, primary the Fock representation is defined by the stationary in-vacuum state $\mid$ in $>$. The transition to the quasiparticle representation for graphene has been executed in [4] with the help of a unitary transformation which can be realized in explicit form. In this representation, the Hamiltonian (2) acquires the diagonal form

$$
H(t)=\int \frac{d^{2} p}{(2 \pi \hbar)^{2}} \varepsilon(\vec{p}, t)\left[a^{+}(\vec{p}, t) a(\vec{p}, t)-b(-\vec{p}, t) b^{+}(-\vec{p}, t)\right]
$$

with the quasienergy $\varepsilon(\vec{p}, t)=V \sqrt{P^{2}(t)}$. The creation and annihilation operators of the quasi-electrons and quasi-holes are defined in the nonstationary vacuum $\mid t>$ and are subject to the standard anticommutation relations. They satisfy the equations of motion [4]

$$
\begin{gathered}
\dot{a}(\vec{p}, t)=\frac{i}{\hbar}[H(t), a(\vec{p}, t)]+i \lambda(\vec{p}, t) b^{+}(-\vec{p}, t), \\
\dot{b}(-\vec{p}, t)=\frac{i}{\hbar}[H(t), b(-\vec{p}, t)]-i \lambda(\vec{p}, t) a^{+}(\vec{p}, t),
\end{gathered}
$$

where the transition amplitude is equal to

$$
\lambda(\vec{p}, t)=\left\{e V^{2} / 2 \varepsilon^{2}\right\}\left[E_{1} P_{2}-E_{2} P_{1}\right]
$$

when exploiting $E(t)=-(1 / c) \dot{A}(t)$.

In the quasiparticle representation, the electron and hole (positron) distribution functions are defined as

$$
\begin{gathered}
f^{e}(\vec{p}, t)=<\operatorname{in}\left|a^{+}(\vec{p}, t) a^{-}(\vec{p}, t)\right| \text { in }>, \\
f^{h}(\vec{p}, t)=<\operatorname{in}\left|b^{+}(-\vec{p}, t) b^{-}(-\vec{p}, t)\right| \text { in }>.
\end{gathered}
$$

Their equality $f^{e}=f^{h}=f$ is ensured by the electroneutrality condition. Using then the standard procedure [3], definitions (6) and equations of motion (4), it is easy to obtain the KE (1). 
It is useful to introduce the auxiliary functions

$$
\left.\begin{array}{l}
u(t) \\
v(t)
\end{array}\right\}=\int_{t^{\prime}}^{t} d t^{\prime} \lambda\left(t^{\prime}\right)\left[1-2 f\left(t^{\prime}\right)\right]\left\{\begin{array}{c}
\cos \theta\left(t, t^{\prime}\right) \\
\sin \theta\left(t, t^{\prime}\right)
\end{array} .\right.
$$

They can be rewritten as the anomalous averages

$$
\begin{aligned}
& f^{(+)}(\vec{p}, t)=<\text { in }\left|a^{+}(\vec{p}, t) b^{+}(-\vec{p}, t)\right| \text { in }>, \\
& f^{(-)}(\vec{p}, t)=<\operatorname{in}\left|b^{-}(-\vec{p}, t) a^{-}(\vec{p}, t)\right| \text { in }>
\end{aligned}
$$

by means of the relations

$$
u=2 \mathfrak{R} f^{(+)}=2 \mathfrak{R} f^{(-)}, \quad v=2 \mathfrak{J} f^{(+)}=-2 \mathfrak{J} f^{(-)} .
$$

These functions describe the vacuum polarization (see below) and are useful in order to write the KE (1) in the form of a system of three ordinary differential equations:

$$
\dot{f}=\frac{1}{2} \lambda u, \quad \dot{u}=\lambda(1-2 f)-2 \varepsilon v, \quad \dot{v}=2 \varepsilon u .
$$

It is natural to select the zero initial conditions $f\left(t_{0}\right)=u\left(t_{0}\right)=v\left(t_{0}\right)=0$. The first equality means the absence of e-h excitations at $t=t_{0}$. The two last equalities can be interpreted as absence of the anomalous correlations (8) at $t=t_{0}$ what corresponds to the in-vacuum state in the case of vacuum QED. These properties can be correlated with the adiabatic hypothesis at $t \rightarrow-\infty$.

However, the opposite asymptotics of the $u$ and $v$ functions are the undamped oscillations after the shutoff of the external field (see below). It corresponds to the long action vacuum Zitterbewegung. It allows to introduce the peculiar time order parameter $\Phi(t)=u(t)+i v(t)$ which satisfies to the equation of motion

$$
\dot{\Phi}-2 i \varepsilon \Phi=\lambda(1-2 f)
$$

The solution of this equation with the zero initial condition is

$$
\Phi(t)=\int_{-\infty}^{t} d t^{\prime} \lambda\left(t^{\prime}\right)\left[1-2 f\left(t^{\prime}\right)\right] \exp \left[2 i \int_{t^{\prime}}^{t} d \tau \varepsilon(\tau)\right] .
$$

Let us consider now a finite electric field which is shut down at the point of time $t_{\text {off }}$, i.e. $E(t>$ $\left.t_{o f f}\right)=0$ and hence $\lambda\left(t>t_{o f f}\right)=0$. Then, for $t>t_{o f f}$ it follows from (12) that

$$
\Phi\left(t>t_{\text {off }}\right)=\Phi_{\text {out }} \exp \left[2 i \varepsilon\left(t-t_{\text {off }}\right)\right]
$$

where the asymptotical value of the quasienergy is equal to

$$
\varepsilon_{\text {out }}=\varepsilon(t \rightarrow \infty)=V\left[\left(\vec{p}-(e / c) \vec{A}_{\text {out }}\right)^{2}\right]^{1 / 2},
$$

$A_{\text {out }}=\lim _{t \rightarrow \infty} A(t)$. The momentum dependent amplitude

$$
\Phi_{\text {out }}(\vec{p})=\int_{-\infty}^{t_{\text {off }}} d t^{\prime} \lambda\left(t^{\prime}\right)\left[1-2 f\left(t^{\prime}\right)\right] \exp \left[2 i \int_{t^{\prime}}^{t_{\text {off }}} d \tau \varepsilon(\tau)\right]
$$

is defined by the time structure of the external field pulse.

The presence of such undamped oscillations of the order parameter is a prerequisite of the discussed effect in the polarized current. 
Thus, $\left|\Phi_{\text {out }}(\vec{p})\right|^{2}=$ const after switching off the external field, i.e. the long-range order is formed. Such situation is typical for a phase transition in systems with broken symmetry. This conclusion follows also from the equation for the modulus of the order parameter

$$
\frac{d}{d t}|\Phi|^{2}=2 \lambda(1-2 f) u
$$

which can be obtained on the basis of (11).

\section{Residual currents}

The current density in the quasiparticle representation can be obtained from the Hamiltonian (2) and consists of conductivity and polarization currents,

$$
\begin{gathered}
j_{k}(t)=c \delta H / \delta A_{k}(t)=j_{k}^{c o n d}(t)+j_{k}^{p o l}(t), \\
j_{k}^{c o n d}(t)=-2 g e c V^{2} \int \frac{d^{2} p}{(2 \pi \hbar)^{2}} \frac{P_{k}}{\varepsilon} f, \\
j_{k}^{p o l}(t)=g e c V^{2} \int \frac{d^{2} p}{(2 \pi \hbar)^{2}} \frac{P_{k}}{\varepsilon} u .
\end{gathered}
$$

Let us note that these formulas are nonperturbative. On the r.h.s. of (18), the vacuum unit in the statistical factor $1+2 f$ drops out. The integration is carried out within the Brillouin zone, and $g=4$ is the degeneracy factor (two Dirac points and two pseudo-spin degrees of freedom).

Now we can investigate the evolution of the currents after shutdown of the external field at $t>t_{\text {off }}$. According to the $\mathrm{KE} \mathrm{(1)} \mathrm{for} t>t_{o f f} \dot{f}(t)=0$, i.e. $f\left(t>t_{o f f}\right)=$ const and the residual conductivity current (18) will be constant. However, the residual polarization current (19) survives according to (13)-(15) and has a nontrivial time dependence,

$$
j_{k}^{p o l}=\left(t>t_{\text {off }}\right)=g e c V^{2} \int \frac{d^{2} p}{(2 \pi \hbar)^{2}} \frac{P_{k}}{\varepsilon} \Phi_{\text {out }} \cos \left[2 \varepsilon_{\text {out }}\left(t-t_{\text {off }}\right)\right] .
$$

The integration over the Brillouin zone leads to a smoothing of the undamped oscillations of the order parameter (13).

For an illustration let us consider a supergaussian electric field model with sharp fronts. figure 1 (left) represents the form of the field pulse

$$
E(t)=E_{0} \exp \left[-\left(t-t_{m}\right)^{n} / 2 \tau^{n}\right], n>2
$$

for $n=4$. The time dependence of the polarization current density during the whole period of evolution is shown on the r.h.s. picture.

From this picture follows that the lifetime $T$ of the residual polarization current in graphene by far exceeds the field pulse duration, $T \gg \tau$.

On a qualitative level the similar picture arises also in $D=2+1$ massless QED with the shortened lifetime in ratio $v_{F} / c \simeq 10^{-2}-10^{-3}$.

It is interesting to note also that in the case of a small coupling constant the polarization current dominates, $\left|j^{\text {pol }}\right| \gg\left|j^{\text {cond }}\right|$ in the leading approximation. However, in the case of the running coupling constant depending on the mean energy (as in QCD) this inequality can be reversed. 

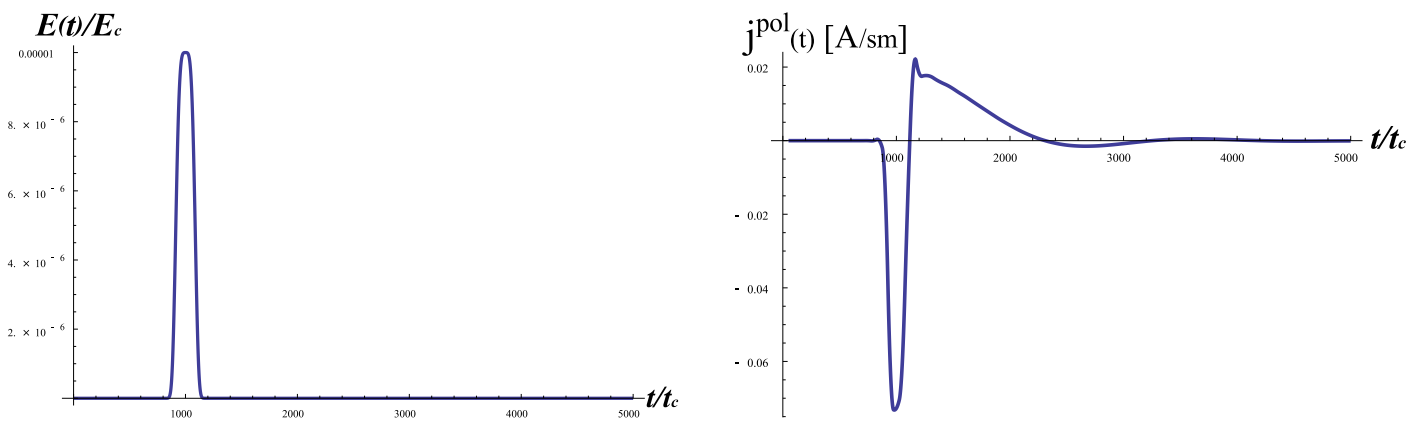

Figure 1. The electric field and the time scale normalized to the characteristic values $E_{c}=\hbar v_{F} / e a^{2}=1.088 \times$ $10^{8} \mathrm{~V} / \mathrm{sm}$ and $t_{c}=a / v_{f}=2.46 \times 10^{-16} \mathrm{~s}(a=2.46 \AA$ is the lattice spacing). Left: Supergaussian electric field (21) $n=4, t_{m}=2.46 \times 10^{-13} s, \tau=2 \times 10^{-14} s$ and $E_{0}=1.088 \times 10^{3} \mathrm{~V} / \mathrm{sm}$. Right: The density of the polarization current.

\section{Conclusion}

The considered vacuum polarization effect is stipulated by inertial properties of the physical quantum vacuum or the vacuumlike state of graphene. In the last case the effect, apparently, can be available for an experimental test on the background of the constant residual conductivity current. However, this potential possibility needs a more detailed investigation of the dependence on external field parameters. On the other hand, it would be interesting to investigate this effect in more realistic massive QED models.

\section{References}

[1] S.A. Smolyansky, A.D. Panferov, D.B. Blaschke, L. Juchnowski, B. Kämpfer, A. Otto, arXiv: 1607.08775 [hep-ph]

[2] A.A. Grib, S.G. Mamaev, V.M. Mostepanenko, Vacuum Quantum Effects in Strong External Fields (Friedmann Laboratory Publishing, St.-Peterburg, 1994)

[3] S.M. Schmidt, D. Blaschke, G. Roepke, S.A. Smolyansky, A.V. Prozorkevich, and V.D. Toneev, Int. J. Mod. Phys. E 7, 709 (1998)

[4] B. Dora, and R. Moessner, Phys. Rev. B 81, 165431 (2010)

[5] H.C. Kao, M. Lewkowicz, and B. Rosenstein, Phys. Rev. B 82, 035406 (2010) 